\title{
Tolerancia a sequía y calor en arroz (Oryza sativa)
}

\section{Drought and heat tolerance in rice (Oryza sativa)}

\author{
Mónica Beatriz López-Hernández ${ }^{1}$, Cándido López-Castañeda ${ }^{1 *}$, Josué Kohashi-Shibata ${ }^{1}$, Salvador \\ Miranda-Colín ${ }^{1}$, Edwin Javier Barrios-Gómez ${ }^{2}$, Carlos Gustavo Martínez-Rueda ${ }^{3}$ \\ ${ }^{1}$ Colegio de Postgraduados, Campus Montecillo, Carretera México-Texcoco km. 36.5, Montecillo, CP. 56230. Municipio de \\ Texcoco, Estado de México, México. \\ ${ }^{2}$ Campo Experimental Zacatepec, INIFAP, Zacatepec, Morelos, México. \\ ${ }^{3}$ Facultad de Ciencias Agrícolas, Universidad Autónoma del Estado de México, El Cerrillo, Piedras Blancas, CP. 50090. Toluca, \\ Estado de México, México. \\ *Autor de correspondencia: clc@colpos.mx
}

Artículo científico recibido: 17 de julio de 2017 aceptado: 02 de mayo 2018

RESUMEN. El objetivo fue determinar la respuesta de genotipos de arroz (Oryza sativa) a la sequía. Se evaluó la temperatura del dosel vegetal (Tc) en emergencia de panícula (EP), floración (F) y en el llenado del grano (PLG), además del efecto de la $T c$ en el rendimiento de grano $(R G)$ y sus componentes bajo riego $(R)$ y sequía $(S)$. El experimento se realizó en el Campo Experimental Zacatepec del Instituto Nacional de Investigaciones Forestales, Agrícolas y Pecuarias. Se evaluaron ocho líneas $F_{6}$ del Fondo Latinoamericano para Arroz de Riego (FLAR) y la variedad nacional El Silverio, bajo un diseño de bloques completos con tres repeticiones en riego y sequía. La siembra se realizó a densidad de 100 $\mathrm{kg} \mathrm{ha}^{-1}$ con dosis de fertilización de 180-40-40 de N, P, K, respecitvamente. La Tc en S fue mayor que en $\mathrm{R}$, también se relacionó de forma negativa con el índice de productividad media (IPM) y el índice de tolerancia a sequía (ITS); las líneas con baja Tc tienen mayor productividad y tolerancia a la sequía. La Tc redujo el RG, biomasa, panículas $\mathrm{m}^{-2}$, granos $\mathrm{m}^{-2}$, granos/panícula y peso de mil granos en un $25,12,13,15,9.6$ y $7 \%$ por cada $1{ }^{\circ} \mathrm{C}$ de incremento en la Tc en R y S. La sequía aumentó la Tc y disminuyó el rendimiento y sus componentes; las líneas con baja Tc redujeron menos su rendimiento y fueron más productivas, y tolerantes a la sequía.

Palabras clave: Biomasa, granos, humedad, rendimiento, temperatura

ABSTRACT. The objective was to determine the response of rice (Oryza sativa) genotypes to drought. Plant canopy temperature $(\mathrm{Ct})$ in panicle emergence $(\mathrm{PE})$, flowering $(\mathrm{F})$ and grain filling $(\mathrm{GF})$ were evaluated, as well as the effect of $\mathrm{Ct}$ on grain yield (GY) and its components under irrigation (I) and drought (D). The experiment was carried out at the Zacatepec Experimental Field operated by the National Institute of Forestry, Agriculture and Livestock Research. Eight $\mathrm{F}_{6}$ lines of the Latin American Fund for Irrigated Rice (FLAR for its acronym in Spanish) and the national variety El Silverio were evaluated under a complete block design with three replicates in irrigation and drought. Planting was carried out at a density of $100 \mathrm{~kg} \mathrm{ha}^{-1}$ with a fertilizer rate of $180-40-40 \mathrm{NPK}$. The $\mathrm{Ct}$ in D was higher than in R, and it was also negatively related to the average productivity index (API) and the drought tolerance index (DTI); the low- $\mathrm{Ct}$ lines have higher productivity and drought tolerance. The $\mathrm{Ct}$ reduced the $\mathrm{GY}$, biomass, panicles $\mathrm{m}^{-2}, \mathrm{GY}$, grain panicle ${ }^{-1}$ and 1000 -grain weight by $25,12,13,15,9.6$ and $7 \%$ for every $1{ }^{\circ} \mathrm{C}$ increase in $\mathrm{Ct}$ in I and $\mathrm{D}$. The drought increased the $\mathrm{Ct}$ and decreased the yield and its components; the low- $\mathrm{Ct}$ lines reduced their yield less and were more productive and drought tolerant.

Key words: Biomass, grains, moisture, yield, temperature 


\section{INTRODUCCIÓN}

El déficit hídrico del suelo o sequía reduce el rendimiento de los cultivos. Con frecuencia la sequía viene acompañada de altas temperaturas o calor que afectan los procesos fisiológicos de la planta como el cierre de estomas, disminución de la transpiración y aumento de la temperatura (Bazzaz et al. 2015). En las regiones tropicales la humedad relativa es una dimensión adicional que puede exacerbar el efecto del estrés por calor en dos formas: (i) el aire saturado reduce el potencial de enfriamiento evaporativo de la planta y (ii) las altas temperaturas nocturnas (Reynolds et al. 2010). Al considerar que las temperaturas óptimas para el crecimiento y desarrollo de diferentes especies cultivadas varían entre $15{ }^{\circ} \mathrm{C}$ para trigo y de 20 a $25{ }^{\circ} \mathrm{C}$ para arroz, maíz, sorgo y soya (Allen et al. 2009), se puede inferir que la temperatura del aire no es un indicador del estrés que experimentan las plantas. Por lo que en ambientes con baja humedad relativa, la temperatura de la planta puede estar algunos grados debajo de la temperatura del aire (Amani et al. 1996). El estrés por calor que experimenta la planta es función de la temperatura del aire y, de los factores genéticos y agronómicos que determinan el potencial de enfriamiento evaporativo de la planta. Los factores genéticos con mayor influencia en el calentamiento de la planta son la cubierta o dosel vegetal, la arquitectura del dosel, la conductancia estomática, la composición de pigmentos, la cera epicuticular, el crecimiento de la raíz, las señales del sistema radical y la fenología; además de factores agronómicos como la fecha de siembra, el método de siembra, riego, sistema de cultivo, manejo de residuos, control de malezas, plagas y enfermedades (Reynolds et al. 2010). Otro factor genético es la esterilidad de las espiguillas en la floración; por lo que la floración al inicio de la mañana, puede ser una estrategia que minimice el daño por calor, característica que puede utilizarse como criterio de selección en los programas de mejoramiento genético del rendimiento en estrés por calor (Bheemanahalli et al. 2017).

Un mayor sistema radical es un carácter adaptativo con buen potencial para incrementar la pro- ductividad en ambientes con sequía y altas temperaturas (Kirkegaard et al. 2007). La medición de las características de crecimiento del sistema radical no es práctica común en los programas de mejoramiento genético, pero medir la temperatura del dosel, permite estimar la cantidad de agua capturada por las raíces. La temperatura del dosel en periodos de estrés extremo está asociada con mayor capacidad de extracción de agua en el perfil del suelo de 60 a $120 \mathrm{~cm}$, durante el llenado del grano (Pask y Reynolds 2013). La temperatura de la planta también sirve para medir la tensión hídrica del suelo, particularmente en las etapas reproductivas del cultivo (Siegfried et al. 2017). La tolerancia al calor junto con otras técnicas pueden ayudar a descubrir los mecanismos que determinan la termotolerancia, lo que podría contribuir a entender las respuestas moleculares al estrés por calor (Sita et al. 2017). Por lo anterior, el presente trabajo tuvo el objetivo de evaluar la respuesta a la sequía y calor de un grupo de genotipos de arroz en las etapas de emergencia de la panícula, antesis y periodo de llenado del grano, en el rendimiento de grano y sus componentes.

\section{MATERIALES Y MÉTODOS}

El experimento se realizó en el Campo Agrícola Experimental del Instituto Nacional de Investigaciones Forestales, Agrícolas y Pecuarias (INIFAP), ubicado en Zacatepec, Morelos, México (18 39' LN, 99 $19^{\prime}$ LO a $900 \mathrm{msnm}$ ) en el ciclo de primavera-verano 2013. La localidad de Zacatepec, se caracteriza por tener un clima cálido subhúmedo con lluvias en verano (Awo (w)), precipitación media de $839 \mathrm{~mm}$ y temperatura media anual de $24.8^{\circ} \mathrm{C}$, con periodo de sequía intraestival o canícula en julio (García 2004). La siembra se realizó en un suelo arcilloso con capacidad de campo de $53.9 \%$, porcentaje de marchitamiento permanente de $31.7 \%$, $\mathrm{pH}$ de 7.5 , conductividad eléctrica de $0.52 \mathrm{dS} \mathrm{m}^{-1}$, materia orgánica de $1.6 \%$, nitrógeno total de $0.9 \%$, fósforo de $9.7 \mathrm{ppm}$ y potasio de $0.22 \mathrm{ppm}$, en el estrato de 0 a $40 \mathrm{~cm}$.

El germoplasma utilizado incluyó ocho líneas 
avanzadas $\left(\mathrm{F}_{6}\right)$ de arroz de alto rendimiento, resistentes al virus de la hoja blanca (Tagosodes oryzicolus) y quema del arroz (Pyricularia oryzae), con calidad de grano superior (Châtel et al. 2010). Las líneas del FLAR tienen un número de días a antesis similar a la variedad testigo El Silverio (Tabla 1). El diseño experimental fue bloques completos al azar en arreglo factorial con tres repeticiones; la unidad experimental fueron seis surcos de $5 \mathrm{~m}$ de longitud separados a $0.2 \mathrm{~m}$. La siembra se realizó en forma directa en suelo seco a chorrillo el 10 de junio de 2013 a densidad de $100 \mathrm{~kg} \mathrm{ha}^{-1}$ y dosis de fertilización de 180-40-40 de N, P y K. Se aplicó una tercera parte del nitrógeno $\mathrm{y}$, todo el fósforo y potasio en la siembra; un segundo tercio de nitrógeno a los 25 días después de la siembra (dds) cuando inició la etapa de amacollamiento y el resto del nitrógeno en la etapa de embuche (70 dds), la fertilización se realizó con urea, superfosfato de calcio triple y cloruro de potasio como fuentes de nitrógeno, fósforo y potasio, respectivamente. Se aplicaron dos tratamientos de humedad edáfica; riego por inundación con la aplicación de agua por gravedad cada semana desde la siembra hasta la madurez fisiológica, para mantener inundado de forma permanente el suelo, y sequía, donde se aplicaron cinco riegos de la siembra a los $26 \mathrm{dds}$, suspendiendo la aplicación de agua hasta la madurez fisiológica de las plantas. La lluvia registrada de la siembra a la madurez fisiológica en el tratamiento de riego fue de $644 \mathrm{~mm}$ y del inicio del tratamiento de sequía (26 dds) a la madurez fisiológica fue de $489 \mathrm{~mm}$. El control de malezas se realizó con dos aplicaciones de herbicida, la primera se realizó en preemergencia tres días después del riego de siembra con Ronstar ${ }^{\circledR}$ a dosis de $2 \mathrm{~L} \mathrm{ha}{ }^{-1}$, para el control de malezas de hoja angosta y la segunda se realizó a los 21 dds cuando las plántulas de arroz tenían de 3 a 4 hojas expuestas, con una mezcla de herbicidas postemergentes Propanil ${ }^{\circledR}$ y $2,4-\mathrm{D}$ diclorofenoxiacético ${ }^{\circledR}$ a dosis de 6.0 y $1.5 \mathrm{~L} \mathrm{ha}^{-1}$, para el control de malezas de hoja angosta (Zacate Johnson y coquillo) y de hoja ancha (Esqueda et al. 2010). El control del barrenador blanco del tallo (Rupela albinella), gusano soldado (Spodoptera frugiperda Smith) y
Chinche café (Oebalus insularis), se realizó con la aplicación de cipermetrina ${ }^{\circledR}$ a dosis de $0.25 \mathrm{~L} \mathrm{ha}^{-1}$ a los 66 y 90 dds.

Las variables medidas en el experimento fueron: la temperatura del dosel vegetal $\left(\mathrm{Tc},{ }^{\circ} \mathrm{C}\right)$ con un termómetro infrarrojo (Modelo Raytek Spectrum Technologies) en un ángulo aproximado de $45^{\circ}$ y distancia de $30 \mathrm{~cm}$ del dosel de las plantas, cada tercer día entre las 12:00 y 15:00 h, de los 58 a los 119 dds (Pietragalla 2013) y la temperatura del aire $\left(\mathrm{Ta},{ }^{\circ} \mathrm{C}\right)$ con un termómetro de máximas y mínimas de columna de mercurio (Brennan) colocado junto a las plantas (Barrios-Gómez et al. 2011).

El rendimiento de grano ( $R G, \mathrm{~g} \mathrm{~m}^{-2}$ ) y sus componentes de biomasa aérea ( $\left.B M, \mathrm{~g} \mathrm{~m}^{-2}\right)$, número de granos $\mathrm{m}^{-2}\left(\mathrm{G} \mathrm{M}^{-2}\right)$, número de panículas $\mathrm{m}^{-2}\left(\mathrm{P} \mathrm{M}^{-2}\right)$, número de granos por panícula (GP) y peso de mil granos (PMG, g) se determinaron después de la madurez fisiológica en todas las plantas. Con los datos del RG se calculó el índice de productividad media (IPM) propuesto por Ashraf et al. (2015), como el promedio del rendimiento en sequía (Ys) y el rendimiento en riego (Yp), con la siguiente ecuación: $I P M=(Y s+Y p) / 2$

El índice de tolerancia a sequía (ITS) propuesto por Ashraf et al. (2015), se calculó como el cociente entre el rendimiento de cada genotipo en sequía (Ys) multiplicado por el cociente entre el rendimiento en sequía (Ys) y el rendimiento en riego (Yp), dividido por el rendimiento promedio de todos los genotipos en riego $(\bar{Y} p)$, con la siguiente ecuación: $\operatorname{ITS}=\left[\frac{Y s\left(\frac{Y s}{Y p}\right)}{Y p}\right]$

Los datos de temperatura máxima y mínima del aire, y precipitación acumulada cada $10 \mathrm{~d}$ durante el estudio, se obtuvieron de una estación meteorológica localizada a $50 \mathrm{~m}$ del experimento, en el Campo Experimental del INIFAP, Zacatepec, Morelos. El contenido de la humedad aprovechable (HA, $\%$ ) del suelo se determinó con una barrena tipo Veihmeyer, en el tratamiento de sequía, cada $10 \mathrm{~d}$ en los estratos de 0 a 20 y 20 a $40 \mathrm{~cm}$, con el método gravimétrico [\% $(\mathrm{HA})=$ (peso de suelo húmedo peso de suelo seco / peso de suelo seco) 100] durante el periodo de estudio. 
Tabla 1. Material genético utilizado en el experimento.

\begin{tabular}{ccccc}
\hline No. de genotipo & Genealogía & Origen $^{\alpha}$ & ${\text { Rendimiento de grano }\left(\mathrm{t} \mathrm{ha}^{-1}\right)}^{\text {Días a antesis }}$ \\
\hline 1 & FL04621-2P-1-3P-3P-M & P-V 2005 & 6.8 & 85 \\
2 & FL05392-3P-12-2P-2P-M & P-V 2006 & 13.2 & 90 \\
3 & FL06747-4P-10-5P-3P-M & P-V 2007 & 10.5 & 89 \\
4 & FL07201-6P-5-3P-3P-M & P-V 2008 & 9.4 & 84 \\
5 & FL08224-3P-2-1P-2P-M & P-V 2009 & 10.9 & 90 \\
6 & FL08378-3P-5-2P-2P-M & P-V 2009 & 11.4 & 87 \\
7 & FL08224-3P-2-1P-3P-M & P-V 2009 & 15.9 & 92 \\
8 & FL10129-12P-4-2P-3P-M & P-V 2011 & 12.1 & 88 \\
9 & El Silverio (Testigo) & P-V 2011 & 12.0 & 91 \\
\hline
\end{tabular}

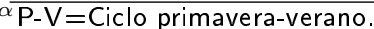

El análisis de varianza y la comparación de medias (DMS, $\leq 0.05)$ para todas las variables se realizó con el programa SAS, Versión 9.4, para Windows.

\section{RESULTADOS}

El análisis estadístico para la temperatura del dosel vegetal $\left(\mathrm{Tc},{ }^{\circ} \mathrm{C}\right)$ y el combinado riego-sequía detectó diferencias $(p \leq 0.01)$ entre genotipos en riego y sequía. Mientras que el análisis combinado riego-sequía detectó diferencias ( $p \leq 0.01$ y $p \leq 0.05)$ entre tratamientos de humedad edáfica y los genotipos en todas las fechas de evaluación. La interacción humedad $x$ genotipos tuvo diferencias $(p \leq 0.01)$ en las fechas de medición, con excepción de las mediciones realizadas a los 66 y 88 dds (Tabla 2). Mientras que el análisis estadístico individual detectó diferencias $(p \leq 0.01)$ para rendimiento de grano, biomasa aérea, número de granos $\mathrm{m}^{-2}$, panículas $\mathrm{m}^{-2}$, granos por panícula, y peso de mil granos en riego y sequía. El análisis combinado riego-sequía detectó diferencias ( $p$ $\leq 0.01$ ) entre tratamientos de humedad y genotipos para rendimiento de grano y todos sus componentes; en tanto que la interacción humedad $x$ genotipos tuvo diferencias ( $p \leq 0.01$ y $p \leq 0.05$ ) para rendimiento de grano, número de granos y panículas $\mathrm{m}^{-2}$, número de granos por panícula, y peso de mil granos. Los índices de productividad media y tolerancia a sequía presentaron diferencias $(p \leq 0.01)$ entre genotipos y la interacción humedad $x$ genotipos (Tabla 2 ).

La temperatura promedio máxima diurna de $33^{\circ} \mathrm{C}$ y mínima nocturna del aire de $17^{\circ} \mathrm{C}$ tuvieron amplia oscilación con promedio de $25{ }^{\circ} \mathrm{C}$ (Figura 1). En tanto que la lluvia se distribuyó de forma irregular con reducciones notorias en la emergencia de la panícula (50 a 60 dds), floración ( 80 a 90 dds) y llenado del grano (110 a 140 dds). La variación en el contenido hídrico del suelo reflejó la disminución en la cantidad de lluvia registrada en la emergencia de la panícula, floración y llenado del grano (Figura 2).

La temperatura del aire $(\mathrm{Ta})$ se relacionó de forma positiva y significativa con la temperatura del dosel vegetal $(T c)$ en riego ( $T c R, r=0.64, p \leq 0.01$ ) y sequía (TcS, $r=0.77, p \leq 0.01$ ) (Figura 3 ). La Tc aumentó $0.8{ }^{\circ} \mathrm{C}$ por cada $1^{\circ} \mathrm{C}$ de incremento en la temperatura del ambiente en riego, mientras que en sequía, la Tc aumentó $1^{\circ} \mathrm{C}$ por cada ${ }^{\circ} \mathrm{C}$ de incremento de la temperatura del aire. La temperatura del dosel vegetal en sequía fue mayor que en riego en las diferentes etapas fenológicas del cultivo; la Tc aumentó con el transcurso del ciclo del cultivo, siendo mayor en el llenado del grano; con incremento mayor en sequía que en riego (Figura 4). Los genotipos 2 y 7 mostraron las menores Tc en emergencia de la panícula, antesis y madurez fisiológica, en riego y sequía; estos genotipos mostraron menor Tc que las demás líneas a pesar del severo estrés hídrico que se desarrolló en la planta, sobre todo en la parte final del periodo de llenado del grano, que coincidió con un periodo de baja precipitación y contenido de humedad en el suelo.

Los índices de productividad media (IPM) y tolerancia a sequía (ITS) se relacionaron de manera negativa con la Tc en promedio de todas las líneas 
Tabla 2. Significancia estadística para la temperatura del dosel vegetal $\left({ }^{\circ} \mathrm{C}\right)$ medida entre los 58 y 119 días después de la siembra (dds), rendimiento de grano y sus componentes, índices de productividad media, tolerancia a sequía en riego y sequía, y promedio riego-sequía.

\begin{tabular}{|c|c|c|c|c|c|c|c|c|}
\hline \multirow[b]{3}{*}{ DDS } & \multirow{2}{*}{\multicolumn{2}{|c|}{ Riego }} & \multirow{2}{*}{\multicolumn{2}{|c|}{ Sequía }} & \multirow{2}{*}{\multicolumn{4}{|c|}{$\begin{array}{c}\text { Factor de variación } \\
\text { Riego-Sequía }\end{array}$}} \\
\hline & & & & & & & & \\
\hline & Genotipos & Bloques & Genotipos & Bloques & Humedad & Bloques & Genotipos & $\begin{array}{c}\text { Interacción humedad } \\
\text { x genotipos }\end{array}$ \\
\hline 58 & $* *$ & ns & $* *$ & ns & $* *$ & ns & $* *$ & $* *$ \\
\hline 62 & ** & ns & ** & ns & ** & ns & ** & ** \\
\hline 66 & ** & ns & ** & ns & ** & ns & ** & Ns \\
\hline 68 & ** & ns & ** & ns & ** & ns & ** & ** \\
\hline 72 & $* *$ & ns & $* *$ & ns & $* *$ & ns & ** & $* *$ \\
\hline 74 & $* *$ & ns & $* *$ & ns & * & ns & $* *$ & $* *$ \\
\hline 76 & $* *$ & ns & ** & ns & * & ns & ** & ** \\
\hline 79 & $* *$ & ns & $* *$ & ns & $* *$ & ns & $* *$ & $* *$ \\
\hline 81 & ** & ns & ** & ns & ** & ns & ** & $* *$ \\
\hline 83 & $* *$ & ns & $* *$ & ns & $* *$ & ns & $* *$ & $* *$ \\
\hline 86 & $* *$ & ns & $* *$ & ns & $* *$ & ns & $* *$ & $* *$ \\
\hline 88 & ** & ns & ** & ns & ** & ns & $* *$ & Ns \\
\hline 90 & ** & ns & $* *$ & ns & $* *$ & ns & $* *$ & $* *$ \\
\hline 95 & $* *$ & ns & $* *$ & ns & $*$ & ns & $* *$ & $* *$ \\
\hline 97 & $* *$ & ns & $* *$ & ns & $* *$ & ns & $* *$ & $* *$ \\
\hline 100 & $* *$ & ns & $* *$ & ns & $* *$ & ns & $*$ & $* *$ \\
\hline 109 & $* *$ & ns & $* *$ & ns & $* *$ & ns & $* *$ & $* *$ \\
\hline 111 & $* *$ & ns & $* *$ & ns & $* *$ & ns & $* *$ & $* *$ \\
\hline 114 & $* *$ & ns & $* *$ & ns & $* *$ & ns & $* *$ & $* *$ \\
\hline 117 & $* *$ & ns & $* *$ & ns & $* *$ & ns & $* *$ & $* *$ \\
\hline 119 & $* *$ & ns & $* *$ & ns & $* *$ & ns & $* *$ & $* *$ \\
\hline RG & $* *$ & ns & $* *$ & ns & $* *$ & ns & $* *$ & $* *$ \\
\hline BM & $* *$ & ns & $* *$ & ns & $* *$ & ns & $* *$ & Ns \\
\hline $\mathrm{G} \mathrm{M}^{-2}$ & $* *$ & ns & $* *$ & ns & $* *$ & ns & $* *$ & $* *$ \\
\hline $\mathrm{P} \mathrm{M}^{-2}$ & $* *$ & ns & $* *$ & ns & $* *$ & ns & $* *$ & $* *$ \\
\hline G P & $* *$ & ns & $* *$ & ns & $* *$ & ns & $*$ & $* *$ \\
\hline PMG & $* *$ & ns & $* *$ & ns & $* *$ & ns & $* *$ & $*$ \\
\hline IPM & - & - & - & - & - & - & $* *$ & $* *$ \\
\hline ITS & - & - & - & - & - & - & $* *$ & $* *$ \\
\hline
\end{tabular}

con IPM $(r=-0.96, p \leq 0.01$, Figura 5a) e ITS $(r=-0.93, p \leq 0.01$, Figura $5 b)$; los genotipos con menor Tc tuvieron mayor índice de productividad media y tolerancia a sequía. Los genotipos 2 y 7 mostraron la más baja Tc y los mayores índices de productividad media y tolerancia a sequía que los otros genotipos. El rendimiento de grano (RG), la biomasa aérea (BM), el número de granos $\mathrm{m}^{-2}$ ( $\mathrm{G}$ $\left.\mathrm{M}^{-2}\right)$, panículas $\mathrm{m}^{-2}\left(\mathrm{P} \mathrm{M}^{-2}\right)$ y granos por panícula $(G P)$, y peso de mil granos (PMG) se asoció de forma negativa con la Tc en riego y sequía. La Tc disminuyó el RG en riego $(r=-0.97, p \leq 0.01$, Figura 6a) y sequía $(r=-0.92, p \leq 0.01)$; la BM en riego $(r=-0.96, p \leq 0.01$, Figura $6 b)$ y sequía $(r=-0.93, p \leq 0.01)$; el número de granos $\mathrm{m}^{-2}$ en riego ( $r=-0.74, p \leq 0.01$, Figura $6 c$ ) y sequía $(r=-0.93, p \leq 0.01)$; el número de panículas $\mathrm{m}^{-2}$ en riego $(r=-0.38, p \leq 0.05$, Figura $6 d)$ y sequía $(r=-0.94, p \leq 0.05)$; el número de granos por panícula en riego $(r=-0.88, p \leq 0.01$, Figura $6 e)$ y sequía $(r=-0.66, p \leq 0.05)$, y el peso de mil granos en riego ( $r=-0.90, p \leq 0.01$, Figura $6 f)$ y sequía $(r=-0.79, p \leq 0.01)$.

En todos los casos, las líneas con mayor Tc tuvieron menor rendimiento de grano, biomasa aérea, número de granos $\mathrm{m}^{-2}$, panículas $\mathrm{m}^{-2}$, granos por panícula, y peso de mil granos. El efecto del incremento en la Tc fue más evidente en el rendimiento de grano, número de granos $\mathrm{m}^{-2}$, panículas $\mathrm{m}^{-2}$, granos por panícula, y peso de mil granos que en 


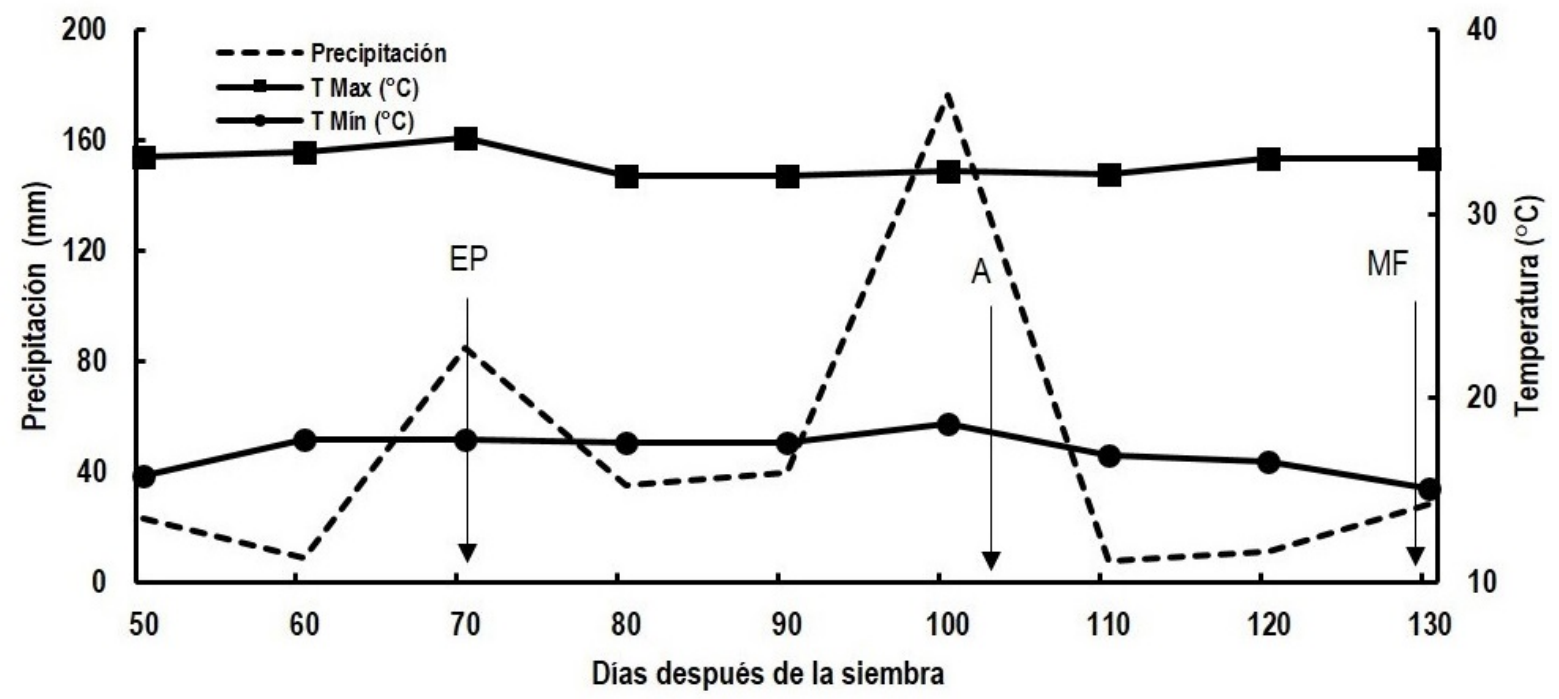

Figura 1. Promedio de temperatura máxima y mínima del aire, y precipitación acumulada cada $10 \mathrm{~d}$, durante el periodo de medición de la temperatura del dosel del cultivo (58-119 dds) en el ciclo primavera-verano. Las flechas indican el promedio del número de días a emergencia de la panícula (EP), antesis (A) y madurez fisiológica (MF).

\section{Dias después de la siembra}

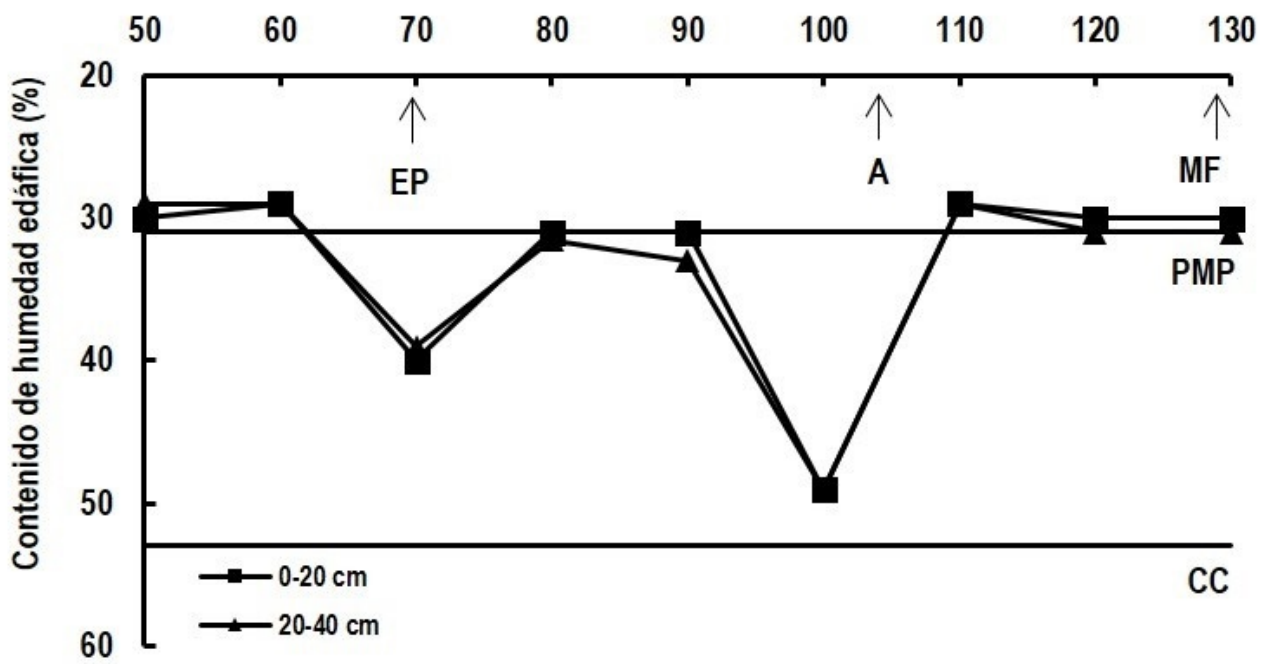

Figura 2. Contenido de humedad edáfica en los estratos de $0-20$ y $20-40 \mathrm{~cm}$ en el tratamiento de sequía durante el periodo de medición de la temperatura del dosel del cultivo (58-119 dds) en el ciclo primavera verano. $\mathrm{PMP}=$ Porcentaje de marchitamiento permanente; $\mathrm{CC}=$ Capacidad de campo. Las flechas indican el promedio del número de días a emergencia de la panícula (EP), antesis (A) y madurez fisiológica (MF).

la biomasa aérea. Los genotipos menos sensitivos a la alta temperatura redujeron el rendimiento y sus componentes en mayor medida que los genotipos menos sensitivos al calor, mostrando los genotipos
2 y 7 la menor temperatura del dosel y reducción del rendimiento de grano, número de granos $\mathrm{m}^{-2}$, panículas $\mathrm{m}^{-2}$, granos por panícula, y peso de mil granos, que los otros genotipos en riego y sequía. En 


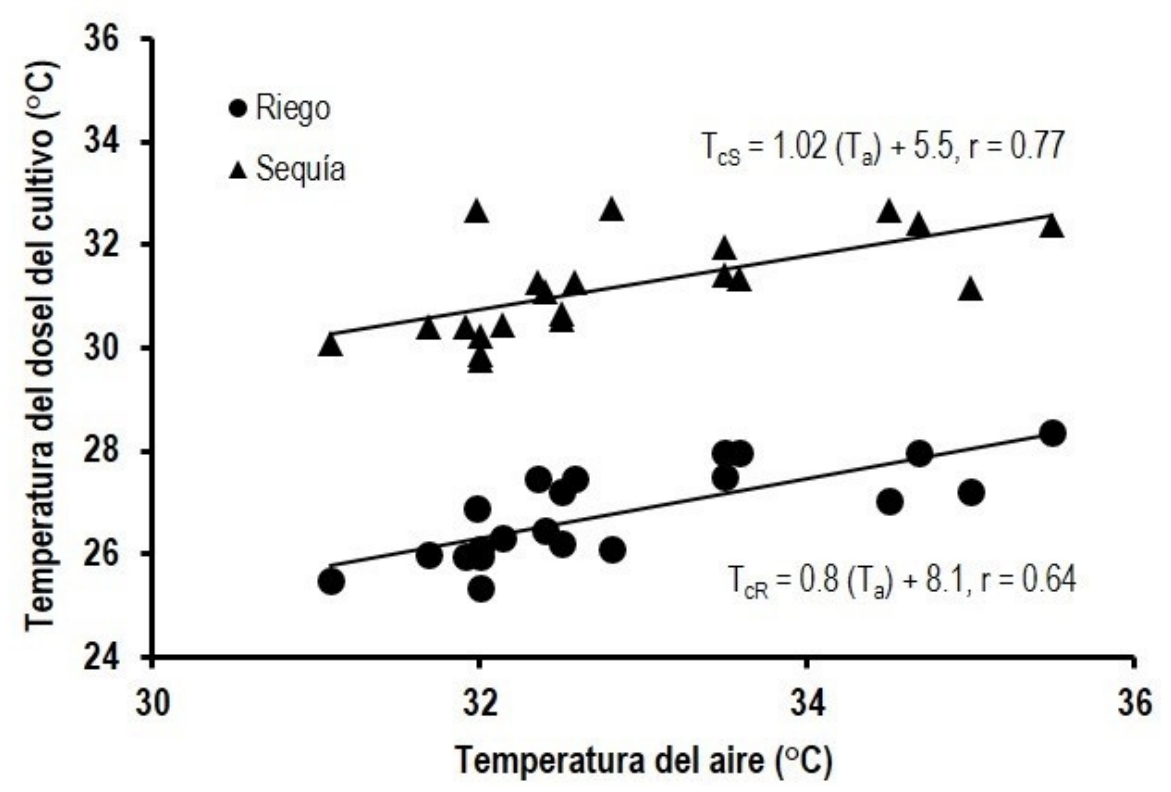

Figura 3. Relación entre temperatura del aire y temperatura del dosel de cultivo en riego y sequía.

tanto que la respuesta en biomasa aérea fue similar en riego y sequía (Figura 6).

Para el rendimiento de grano y sus componentes de cada genotipo expresado como porcentaje con respecto a su media general en riego y sequía, mostró que la mayor variación entre genotipos se tuvo para el rendimiento de grano, la biomasa aérea y el número de granos $\mathrm{m}^{-2}$ y panículas $\mathrm{m}^{-2}$. El número de granos por panícula y peso de mil granos mostraron menor variabilidad entre genotipos. La alta Tc disminuyó el rendimiento de grano, la biomasa aérea, los granos $\mathrm{m}^{-2}$, las panículas $\mathrm{m}^{-2}$, los granos por panícula y el peso de mil granos en un $25,12,25,13,9.6$ y $7 \%$ por cada $1{ }^{\circ} \mathrm{C}$ de incremento en la temperatura del dosel vegetal en los genotipos en riego y sequía. Los genotipos 2 y 7 mostraron menor reducción en el rendimiento de grano y sus componentes que los demás genotipos por efecto de la temperatura alta en riego y sequía.

\section{DISCUSIÓN}

Las deficiencias de agua en el suelo en las etapas de emergencia de la panícula, antesis y llenado del grano en combinación con altas temperaturas máximas promedio de $33{ }^{\circ} \mathrm{C}$ del aire durante el día, aumentaron la temperatura del dosel de las plantas de arroz, en las plantas sometidas a déficit hídrico. La temperatura del dosel vegetal en sequía alcanzó valores de 4 a $5{ }^{\circ} \mathrm{C}$ más altos que en riego en la emergencia de la panícula y antesis, y subió de 28 a $35.5{ }^{\circ} \mathrm{C}$ durante el periodo de llenado del grano. El estrés por calor en condiciones de déficit hídrico durante la floración y el llenado del grano causa esterilidad del polen, deshidratación de los tejidos, daño oxidativo en los cloroplastos y aumento en la fotorespiración (Farooq et al. 2009). Además, las altas temperaturas en presencia de deficiencias hídricas reducen el índice y duración del área foliar, lo que se refleja en disminución de biomasa y rendimiento de grano (Cossani y Reynolds 2012).

El incremento en la temperatura del aire circundante a las plantas entre la emergencia de la panícula y el periodo de llenado del grano tuvo mayor efecto en la temperatura del dosel vegetal de las plantas bajo sequía que en riego. Se observó que las líneas 2 y 7 tuvieron menor temperatura del dosel vegetal que las otras líneas, en condiciones de déficit 


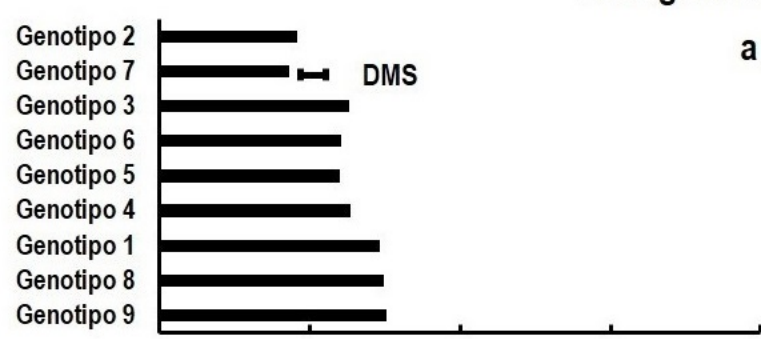

\section{Emergencia de la panícula}

\section{Genotipo 2 \\ Genotipo 3 \\ Genotipo 6 \\ Genotipo 5 \\ Genotipo 4 \\ Genotipo 1 \\ Genotipo 8}

Genotipo 9

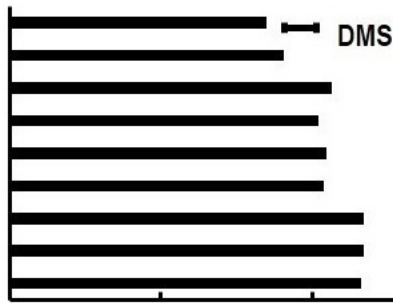

Antesis

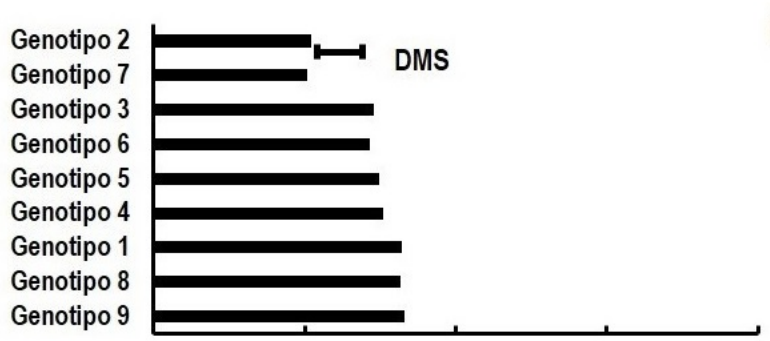

b

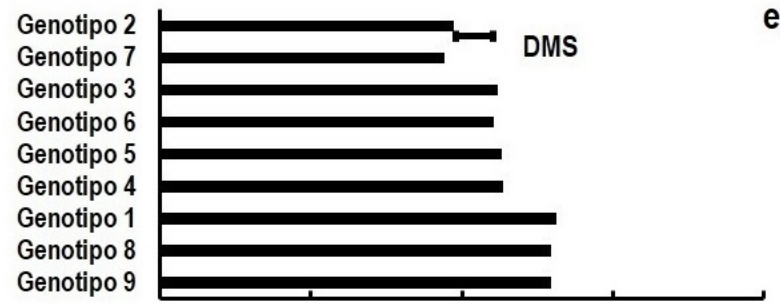

Periodo de llenado de arano

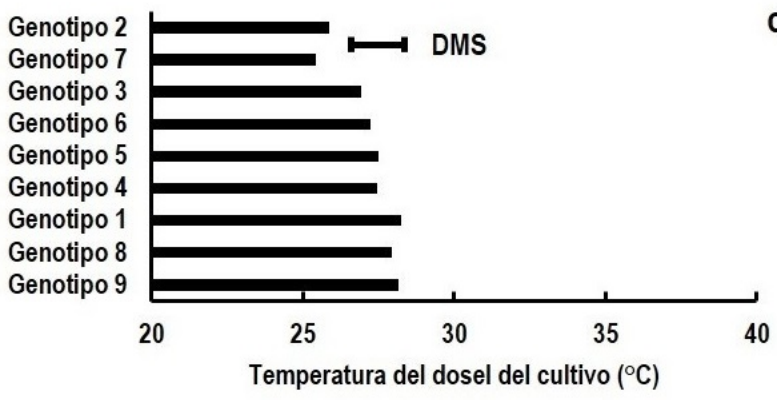

C

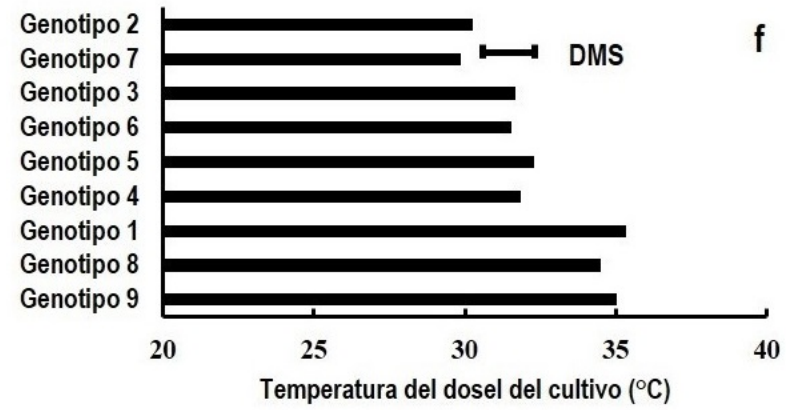

Figura 4. Temperatura del dosel del cultivo $\left({ }^{\circ} \mathrm{C}\right)$ en emergencia de la panícula, antesis y periodo del llenado del grano en riego (a, b y c) y sequía ( $d$, e y $f$ ). La barra horizontal representa el valor de la diferencia mínima significativa $(p \leq 0.05)$.

hídrico, lo que se podría interpretar como respuesta de tolerancia a calor. La expresión de atributos de tolerancia a calor, tolerancia a sequía y tolerancia combinada durante la diferenciación del meristemo floral juega un papel crucial para reducir el daño durante la floración y otras etapas sensitivas de la planta (Jagadish et al. 2015).

La temperatura del dosel vegetal se relaciona con el estrés hídrico de la planta por lo que bajas temperaturas de la planta indican alto potencial hídrico y osmótico de la hoja y viceversa, altas temperaturas de la planta se asocian con bajos potenciales hídrico y osmótico de la hoja (BarriosGómez et al. 2011). El aumento en la temperatura del dosel del cultivo disminuyó el índice de productividad media y el índice de tolerancia a sequía; mostrando las líneas 2 y 7 los mayores índices de productividad media y tolerancia a sequía con menor temperatura del dosel vegetal que las otras líneas. Estas líneas tuvieron menor pérdida de rendimiento de grano, menor grado de estrés y dosel vegetal con menor temperatura al medio día. La respuesta diferencial entre la temperatura de las plantas en riego y las plantas en sequía, se debe a que las 

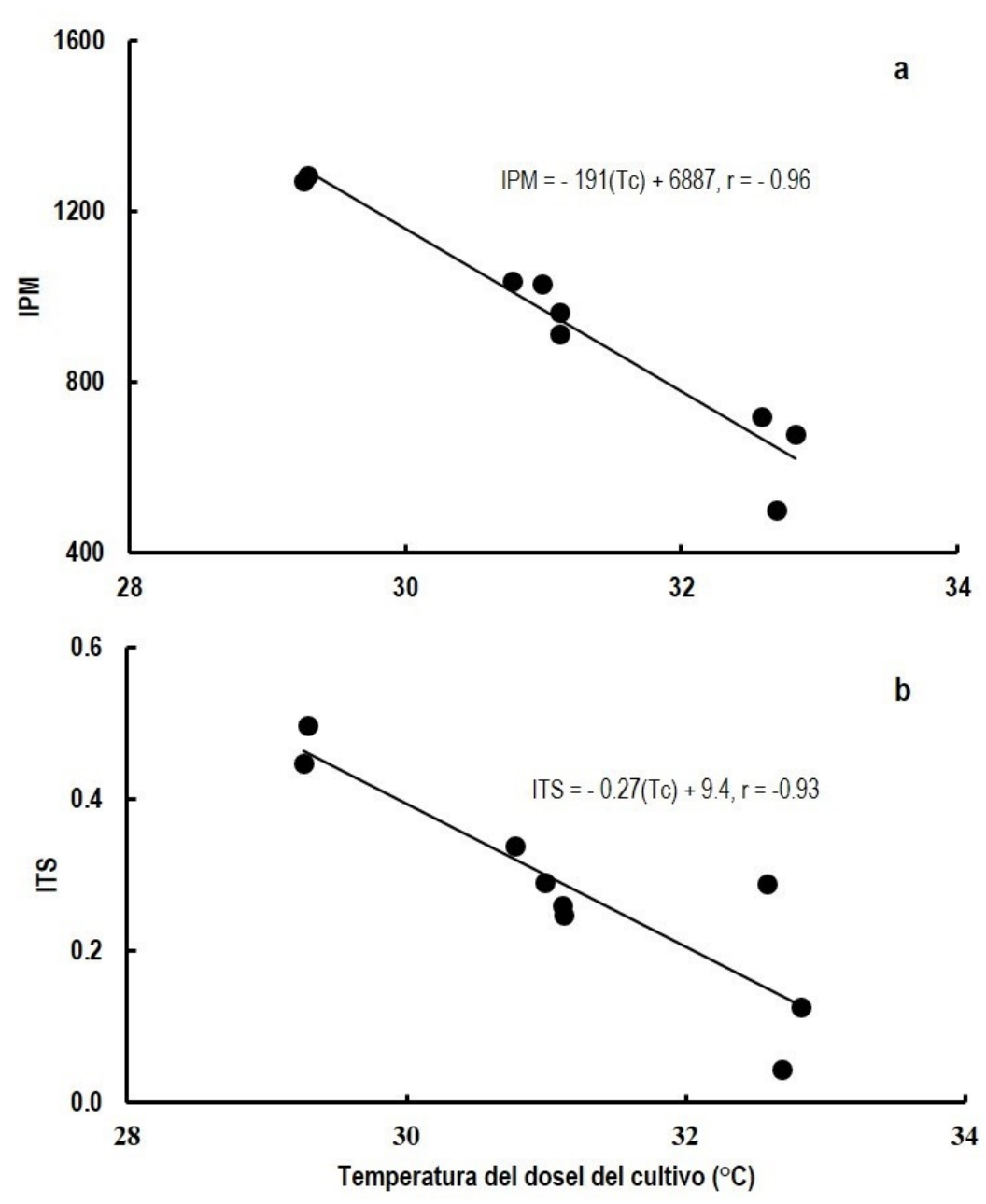

Figura 5. Relación entre la temperatura del dosel del cultivo y el índice de productividad media (IPM) (a) y el índice de tolerancia a sequía (ITS) (b), para rendimiento de grano en promedio de todos los genotipos de arroz.

plantas en condiciones de déficit de agua sufrieron estrés hídrico, por la reducción en el enfriamiento transpiracional de la hoja a consecuencia del cierre estomático (Pinter et al. 1990). La pérdida de turgencia en la planta influye en la velocidad de apertura y cierre estomático en condiciones de estrés; el retraso en el cierre de estomas afecta de forma negativa la eficiencia en el uso del agua, diámetro del tallo, peso del grano por tallo y fecha de espigamiento ( $\mathrm{Qu}$ et al. 2016).

En la madurez fisiológica las características más afectadas por el déficit hídrico del suelo y la alta temperatura del aire durante la emergencia de la panícula, la floración y el llenado del grano fueron el rendimiento de grano, número de panículas $\mathrm{m}^{-2}$, granos $\mathrm{m}^{-2}$, granos por panícula, y el peso de mil granos. El déficit hídrico durante la emergencia de la panícula y la floración afectó más a la longitud del pedúnculo que al porcentaje de espiguillas fértiles, mientras que el calor tuvo mayor efecto en la fertilidad de las espiguillas que en la longitud del pedúnculo; pero el efecto combinado de ambos factores fue mayor que el efecto por separado. En general la fertilidad de las espiguillas fue más afectada que la longitud del pedúnculo (Jagadish et al. 2011, Jagadish et al. 2015, Yang et al. 2017). La 

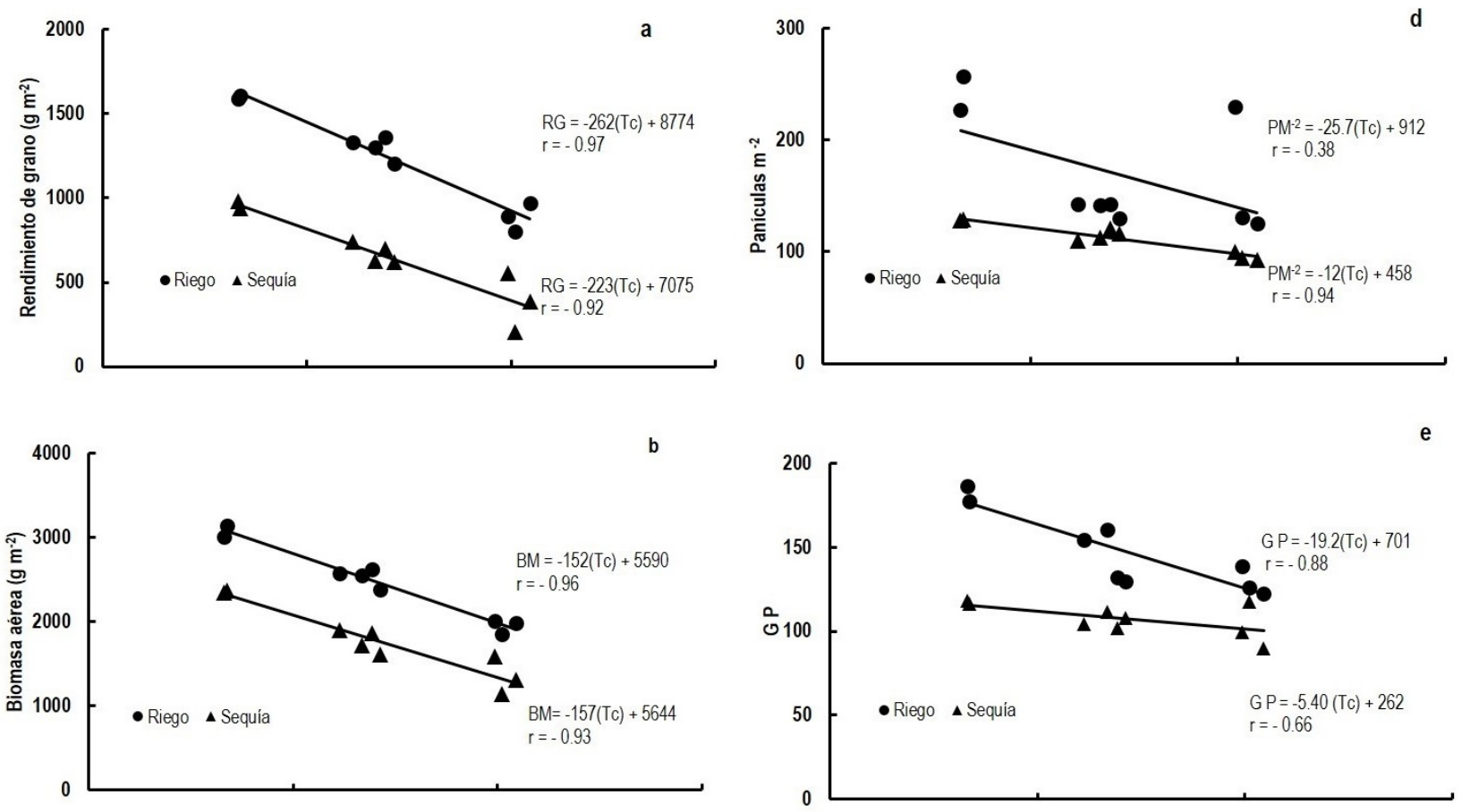

f
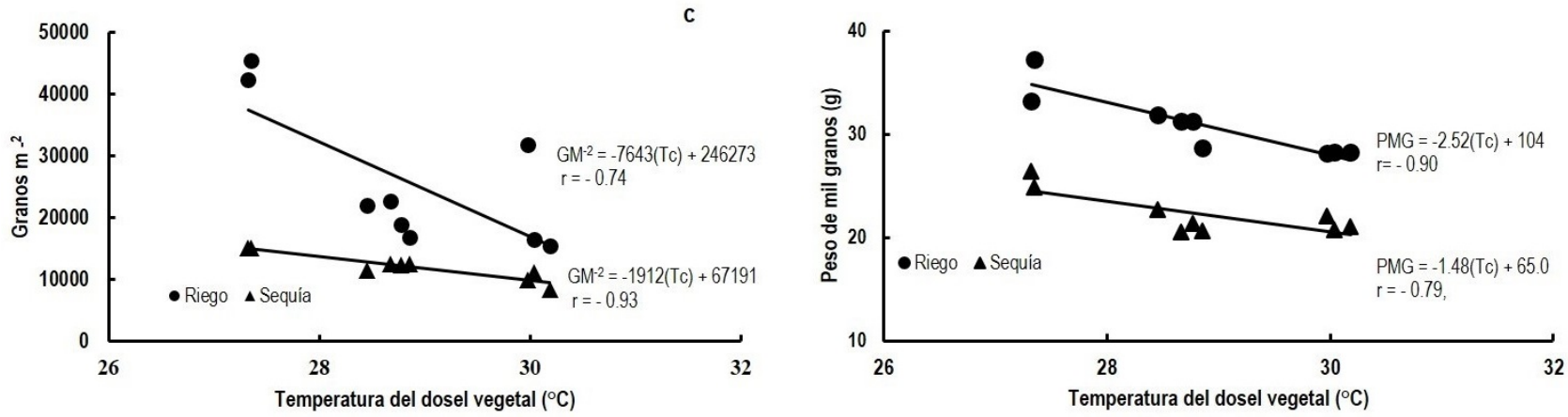

Figura 6. Relación entre la temperatura del dosel del cultivo $\left({ }^{\circ} \mathrm{C}\right)$ promediada de los 58 a los 119 dds y el rendimiento de grano (a), biomasa aérea (b), granos $\mathrm{m}^{-2}$ (c), panículas $\mathrm{m}^{-2}$ (d), granos por panícula (G P) y peso de mil granos (PMG) en riego y sequía.

disminución en el número de espiguillas fértiles y el rendimiento de grano en condiciones de estrés por calor se debe a la reducción en la tasa de fertilización al inicio de la formación del grano y el acortamiento del periodo de llenado del grano, lo que disminuyó la masa del grano y estructura del endospermo (Cao et al. 2016).

El estrés hídrico combinado con estrés por calor durante la emergencia de la panícula y la floración en arroz, redujo la elongación del pedúnculo y la fertilidad de las espiguillas; en estas condiciones de estrés ambiental, la selección para mayor número de espiguillas por panícula y peso de mil granos pueden contribuir a incrementar el rendimiento de grano (Bathia 2017). Las líneas 2 y 7 tuvieron mayor rendimiento de grano, biomasa aérea, número 


\begin{tabular}{|c|c|c|c|c|c|c|}
\hline No. de genotipo & $\begin{array}{c}\text { RG } \\
(\%)\end{array}$ & BM & $\mathrm{G} \mathrm{M}^{-2}$ & $\mathrm{PM}^{-2}$ & G P & PMG \\
\hline 2 & 136 & 122 & 160 & 137 & 115 & 117 \\
\hline 7 & 138 & 119 & 152 & 126 & 119 & 109 \\
\hline 3 & 110 & 99 & 83 & 94 & 91 & 96 \\
\hline 6 & 111 & 103 & 89 & 90 & 101 & 102 \\
\hline 5 & 103 & 100 & 93 & 90 & 106 & 96 \\
\hline 4 & 98 & 92 & 78 & 87 & 93 & 93 \\
\hline 1 & 73 & 83 & 63 & 77 & 83 & 91 \\
\hline 8 & 77 & 92 & 110 & 117 & 93 & 93 \\
\hline 9 El Silverio (Testigo) & 54 & 91 & 73 & 80 & 95 & 91 \\
\hline Media general $\beta$ & 934 & 1185 & 18928 & 141 & 128 & 27 \\
\hline
\end{tabular}

de panículas $\mathrm{m}^{-2}$, granos $\mathrm{m}^{-2}$, granos por panícula, y peso de mil granos que las otras líneas, lo que confirma su superioridad en tolerancia a sequía y calor. Entre estos componentes del rendimiento, el número de espiguillas por panícula y el peso de mil granos podrían utilizarse en la selección para aumentar el rendimiento de grano en plantas de arroz bajo condiciones de déficit hídrico.

La temperatura durante la noche redujo la fertilidad de las espiguillas y el peso del grano por panícula. Al respecto Coast et al. (2014) indican que en estas condiciones de estrés las características más útiles son: las espiguillas con baja temperatura, espiguillas con alta fertilidad, floración precoz y corta duración de la antesis. Las etapas de embuche y floración son las más sensitivas a altas temperaturas, lo que puede llevar a la planta a esterilidad; por lo que la floración en las horas con menor temperatura del día, viabilidad del polen, anteras grandes, dehiscencia basal y la presencia de poros basales grandes son de las características fenotípicas que podrían utilizarse (Bheemanahalli et al. 2017). El efecto del calor durante la floración es más severo en las espiguillas inferiores de la panícula, donde la temperatura disminuye la tasa de fertilización, acelera el llenado del grano y reduce la duración del periodo de llenado, lo que resulta en granos y endospermo más pequeños (Cao et al. 2016).

Las líneas sobresalientes 2 y 7 tuvieron mayor rendimiento de grano, biomasa aérea, granos $\mathrm{m}^{-2}$, panículas $\mathrm{m}^{-2}$, granos/panícula y peso de mil granos que las otras líneas en riego y sequía. Lo que sugiere que el mejor comportamiento agronómico de estas líneas se debe a mayor tolerancia a sequía y calor, que les permiten adaptación a las condiciones de estrés hídrico y calor. Otros estudios reportan que un sistema radical mayor, puede capturar más agua del suelo (Gowda et al. 2011, Kijoji et al. 2014) y mantener un dosel con menor temperatura en condiciones de déficit hídrico en el suelo, en las etapas reproductivas del cultivo (Siegfried et al. 2017).

\section{CONCLUSIONES}

La temperatura del aire se relacionó con la temperatura del dosel vegetal en riego y sequía, la temperatura del dosel vegetal en condiciones de déficit hídrico fue mayor que en riego y en las etapas de antesis y llenado del grano que en emergencia de la panícula. Los genotipos con menor temperatura del dosel vegetal tuvieron mayor índice de productividad media e índice de tolerancia a sequía. El rendimiento de grano, biomasa aérea, número de granos $\mathrm{m}^{-2}$, panículas $\mathrm{m}^{-2}$, granos por panícula, y peso de mil granos disminuyó en $25,12,25,13,9.6$ y $7 \%$ por cada $1{ }^{\circ} \mathrm{C}$ de incremento de la temperatura del dosel vegetal en riego y sequía. Las líneas 2 y 7 tuvieron menor temperatura del dosel vegetal desde la emergencia de la panícula hasta el periodo de llenado del grano en riego y sequía; la menor tem- 
peratura del dosel se reflejó en mayores índices de productividad y tolerancia a sequía. La medición de la temperatura del dosel vegetal podría integrarse en los programas de mejoramiento genético, para seleccionar genotipos tolerantes al estrés hídrico y altas temperaturas.

\section{LITERATURA CITADA}

Allen CD, Birkeland C, Chapin III FS, Groffman PM, Guntenspergen GR, Knapp AK, et al. (2009) Examples of threshold change in ecosystems. In: Charles CW (ed) Threshold of climate change in ecosystems. U.S. Climate Change Science Program and the Subcommittee on Global Change Research. Washington, USA. pp: $35-40$.

Amani I, Fischer RA, Reynolds MP (1996) Canopy temperature depression association with yield of irrigated spring wheat cultivars in a hot climate. Journal of Agronomy and Crop Science 176: 119-129.

Ashraf A, El-Mohsen A, El-Shafi MAA, Gheith EMS, Suleiman HS (2015) Using different statistical procedures for evaluating drought tolerance indices of bread wheat genotypes. Advance in Agriculture and Biology 4: $19-30$.

Barrios-Gómez EJ, López-Castañeda C, Kohashi-Shibata J (2011) Relaciones hídricas y temperaturas altas en frijol del tipo "Flor de Mayo". Agronomía Costarricense 35: 131-145.

Bathia D, Joshi S, Das A, Vikal Y, Sahi GK, Neelam K, et al. (2017) Introgression of yield component traits in rice (Oryza sativa ssp. indica) through interspecific hybridization. Crop Science 57: 1557-1573.

Bazzaz MdM, Khaliq QA, Karim MdA, Al-Mahmud A, Khan MdSA (2015) Canopy temperature and yield based selection of wheat genotypes for water deficit environment. Open Access Library Journal 2: 1-11.

Bheemanahalli R, Sathishraj R, Manoharan M, Sumanth HN, Muthurajan R, Ishimaru T, et al. (2017) Is early morning flowering an effective trait to minimize heat stress damage during flowering in rice? Field Crops Research 203: 238-242.

Cao YY, Chen YH, Chen MX, Wang ZQ, Wu CF, Bian XC, et al. (2016) Growth characteristics and endosperm structure of superior and inferior spikelets of indica rice under high-temperature stress. Biologia Plantarum 60: 532-542.

Châtel M, Guimarães EP, Ospina Y, Rodríguez F, Lozano VH (2010) Mejoramiento de poblaciones de arroz de secano empleando selección recurrente y desarrollo de variedades. En: Degiovanni BV, Martínez RCP, Motta OF (ed) Producción eco-eficiente del arroz en América Latina. Centro Internacional de Agricultura Tropical. Publicación CIAT No. 370. Cali, Colombia. pp: 207-224.

Coast O, Ellis RH, Murdoch AJ, Quiñones C, Jagadish KSV (2014) High night temperature induces contrasting responses for spikelet fertility, spikelet tissue temperature, flowering characteristics and grain quality in rice. Functional Plant Biology 42: 149-161.

Cossani CM, Reynolds MP (2012) Physiological traits for improving heat tolerance in wheat. Plant Physiology 160: $1710-1718$.

Esqueda E VA, Tosqui VOH, Flores MDS (2010) Control de malezas en el cultivo del arroz de temporal en Veracruz. Campo Experimental Cotaxtla, Centro de Investigación Regional Golfo Centro, INIFAP. Folleto Técnico No. 53. Medellín de Bravo, Veracruz, México. 41p.

Farooq M, Wahid A, Kobayashi N, Fujita D, Basra SMA (2009) Plant drought stress: effects, mechanisms and management. Agronomy for Sustainable Development 29: 185-212. 
García E (2004) Modificaciones al sistema de clasificación climática de Köppen. Quinta edición. Instituto de Geografía, UNAM. México. 217p.

Gowda VRP, Henry A, Yamauchi A, Shashidhar HE, Serraj R (2011) Root biology and genetic improvement for drought avoidance in rice. Field Crops Research 122: 1-13.

Jagadish SVK, Muthurajan R, Rang ZW, Malo R, Heuer S, Bennett J, et al. (2011) Spikelet proteomic response to combined water deficit and heat stress in rice (Oryza sativa cv. N22). Rice 4: 1-11.

Jagadish SVK, Murty MVR, Quick WP (2015) Rice responses to rising temperatures? challenges, perspectives and future directions. Plant, Cell and Environment 38: 1686-1698.

Kijoji AA, Nchimbi-Msolla S, Kanyeka ZL, Serraj R, Henry A (2014) Linking root traits and grain yield for rainfed rice in sub-Saharan Africa: Response of Oryza sativa x Oryza glaberrima introgression lines under drought. Field Crops Research 165: 25-35.

Kirkegaard JA, Lilley JM, Howe GN, Graham JM (2007) Impact of subsoil water use on wheat yield. Australian Journal of Agricultural Research 58: 303-315.

Pask AJD, Reynolds MP (2013) Breeding for yield potential has increased deep soil water extraction capacity in irrigated wheat. Crop Science 53: 2090-2104.

Pietragalla, J (2013) Temperatura del dosel. En: Pask AJD, Pietragalla J, Mullan DM, Chávez-Dulanto PN, Reynolds MP (ed). Fitomejoramiento fisiológico II: Una guía de campo para la caracterización fenotípica de trigo. CIMMYT. México. pp: 10-14

Pinter Jr PJ, Zipoli G, Reginato RJ, Jackson RD, Idso SB, Hohman JP (1990) Canopy temperature as an indicator of differential water use and yield performance among wheat cultivars. Agricultural Water Management 18: 35-48.

Qu M, Hamdani S, Li W, Wang S, Tang J, Chen Z, et al. (2016) Rapid stomatal response to fluctuating light: An under explored mechanism to improve drought tolerance in rice. Functional Plant Biology 43: 727-738.

Reynolds MP, Hays D, Chapman S (2010) Breeding adaptation to heat and drought stress. In: Reynolds MP (ed). Climate change and crop production. CAB International, CPI Group. UK. pp: 71-91.

Siegfried J, Khosla R, Longchamps L (2017) Infrared thermometry to quantify in-field soil moisture variability. Journal of Crop Improvement 31: 72-90.

Sita K, Sehgal A, HanumanthaRao B, Nair RM, Vara PV, Kumar S, et al. (2017) Food legumes and rising temperatures: Effects, adaptive functional mechanisms specific to reproductive growth stage and strategies to improve heat tolerance. Frontiers in Plant Science 8. Article 1658. DOI:1033389/fpls.2017.01658.

Yang Z, Zhang Z, Zhang T, Fahad S, Cui K, Nie L, Peng S Huang J (2017) The effect of season-long temperature increases on rice cultivars grown in the central and southern regions of China. Frontiers in Plant Science 8. Article 1908. DOI:10.3389/fpls.2017.01908. 
